\title{
Assessment of the concrete strength in existing buildings using a finite population approach
}

\author{
Nuno Pereira, Xavier Romão* \\ Civil Engineering Department, Faculdade de Engenharia da Universidade do Porto, Rua Dr. Roberto Frias, 4200-465 Porto, Portugal
}

\section{H I G H L I G H T S}

- A new method to assess concrete strength in existing building is presented.

- The method disaggregates the concrete variability into finite populations.

- The $\mathrm{CoV}$ of the concrete strength is evaluated using the $\mathrm{CoV}$ of rebound hammer tests.

- The method controls the uncertainty in the estimate of concrete strength variability.

- The method controls the uncertainty in the estimate of the mean concrete strength.

\section{A R T I C L E I N F O}

Article history:

Received 21 September 2015

Received in revised form 2 February 2016

Accepted 5 February 2016

\section{Keywords:}

Concrete strength

Uncertainty

Finite population

Non-destructive tests

RC buildings

\begin{abstract}
A B S T R A C T
A framework is defined to evaluate the concrete compressive strength in existing buildings and control the uncertainty associated to the survey planning and to the concrete strength randomness. The framework proposes the discretization and disaggregation of the concrete strength in a building into finite populations of elements. Finite population statistics are used to correlate the number of tests performed in each population with the uncertainty about the mean and the coefficient of variation ( $\mathrm{CoV})$ of the concrete strength. A method to estimate the $\mathrm{CoV}$ of the concrete strength using the $\mathrm{CoV}$ of rebound hammer test results is also proposed to overcome the need for a high number of destructive tests. Results show that the proposed approach effectively controls the uncertainty in the estimate of the variability of the concrete strength in a population as well as the uncertainty in the estimate of the mean value of the concrete strength.
\end{abstract}

(c) 2016 Elsevier Ltd. All rights reserved.

\section{Introduction}

In the safety assessment of existing buildings, quantifying the "as-built" material properties is of the utmost importance due to the impact that it has on the subsequent application of safety assessment methods. In the case of reinforced concrete (RC) buildings, the concrete compressive strength is a material property that requires careful consideration [1] due to its inherent variability. This fact leads to the usual consideration of the concrete strength as being a random variable that has a certain (unknown) level of aleatory uncertainty [2]. This aleatory uncertainty is related to the inherent variability of the hardened concrete strength in existing structures [3] which can reach large values [4,5], often exceeding a coefficient of variation (CoV) of 20\% [6]. Among other factors, this variability is associated with mix, casting and curing

\footnotetext{
* Corresponding author.

E-mail address: xnr@fe.up.pt (X. Romão).
}

operations, which require a significant level of workmanship. Several studies (e.g. see $[3,7,8]$ ) have analyzed the impact of workmanship on the strength of hardened concrete and found that it can induce several types of variability depending on the structural system being analyzed. Primarily, expected variations can be associated to batch-to-batch variability, involving the randomness related mainly with the construction management and planning and with quality control. Likewise, member-to-member variability can occur due to the influence of workmanship in casting operations. Variations of the concrete strength can also be expected within each structural member due to the previously mentioned factors. Moreover, a recent study [9] also described cracking, damage and the selection of the testing positions within the length of a structural element as sources of potential variability.

In addition to the aleatory uncertainty associated with the concrete strength, epistemic uncertainty will also be generated due to the lack of knowledge associated with non-surveyed structural elements. Since survey plans only comprise tests on a 
few structural members in order to minimize the damage and the cost of inspection operations, the selection of a given set of elements to be tested instead of another will generate uncertainty. This uncertainty is even more important due to the low number of material tests that are generally carried out in existing buildings, a trend partially supported by existing norms (e.g. [10-13]). Often, standards regulating the assessment of existing buildings require a limited number of tests/inspections to be performed at each storey and for each type of primary component that is part of the building in order to obtain estimates of the mean values of the material properties. Nonetheless, as referred in [14], current building codes do not address the uncertainty level in the survey results and neglect the impact that sampling may have on the estimate of the dispersion of concrete strength (specifically on the estimate of the (oV) and on the corresponding estimate of the mean value. Therefore, controlling the epistemic uncertainty about the CoV of the concrete strength is a key component of a survey framework since it will affect the variability of the estimate (i.e. its precision), especially when it is based on a reduced number of tests. Moreover, this uncertainty is also seen to depend on the relation between the number of structural elements that are not tested during survey operations and the total number of structural elements of the population.

To control the extent of this uncertainty in survey operations and its impact on the estimate of the mean value of the concrete compressive strength in existing buildings, a method based on finite population statistics is proposed herein. The proposed approach will enable to effectively control the uncertainty in the estimates of the variability and of the mean value of the concrete strength in a population to improve their reliability. By accounting for the number of structural elements that are not tested during survey operations, the proposed method overcomes limitations of current standard methods and enables the development of more consistent survey frameworks to assess concrete strength in existing buildings.

\section{Assessing statistical parameters in finite populations}

In statistics, a population is said to be finite when it is possible to count all its elements. Statistical parameters characterizing these populations have specific features which are associated to finite size conditions. To evaluate the exact value of these parameters, knowledge about all the $N$ independent elements of the population is required. If all the $N$ elements are observed, the population mean is then:

$\bar{x}_{U}=\frac{1}{N} \cdot \sum_{k=1}^{N} x_{k}$

where $U$ represents the population, $N$ is the finite population size and $x_{k}$ is an individual element of $U$. By the same principles, the variance of the population is given by:

$S_{U}=\frac{1}{N-1} \cdot \sum_{k=1}^{N}\left(x_{k}-\bar{x}_{U}\right)^{2}$

If instead of observing all the $N$ elements of the finite population, a sample with size $n(n<N)$ is observed, estimates for $\bar{x}_{U}$ and $S_{U}$ can be computed. Assuming a simple random sampling of $n$ elements without replacement from an unordered population of size $N, M$ combinations of $n$ elements can be defined, with $\mathrm{M}$ being given by:

$M=\left(\begin{array}{c}N \\ n\end{array}\right)=\frac{N !}{n !(N-n) !}$

The main characteristic of finite population statistics resides in the conditional correlation between the probabilities of observing different values that is introduced by sampling. In finite popula- tions, increasing the sample size $n$ will affect the estimates of the statistical parameters since the observation of element $x_{k}$ will affect the probability of observing the next element in the sample, i.e. $x_{k+1}$. This fact leads to sampling probabilities that depend on $n$, thus reducing the level of statistical uncertainty (that is implicit when considering a sample to represent the population) in the estimators for the statistical parameters when compared to that of infinite populations.

In a finite population with $N$ elements, an estimate $\hat{\bar{x}}_{U}$ for the real mean $\bar{x}_{U}$ obtained using a sample with $n$ elements is defined by:

$\hat{\bar{x}}_{U}=\frac{1}{n} \cdot \sum_{k=1}^{n} x_{k}$

The theoretical variance of the estimator $\hat{\bar{\chi}}_{U}$ obtained with a sample of $n$ elements is defined by:

$S\left(\hat{\bar{x}}_{U}\right)=\frac{1}{n} \cdot\left(\frac{N-n}{N-1}\right) \cdot S_{U}$

where $\left(\frac{N-n}{N-1}\right)$ is the squared value of the finite population correction factor [15]. Based on Eq. (5), the variance of the estimate of the mean can be seen to converge to zero as $n$ converges to $N$, which implies that the sample mean will converge to the true population mean at a rate given by the finite population correction factor. Therefore, this factor is seen as a representation of the statistical uncertainty in the estimate for the finite population mean. Still, in a general case where $n<N$, the variance of the estimate of the mean will be a direct function of $S_{U}$, thus showing the importance of knowing the variability of the concrete strength in order to control the uncertainty in the estimate of the mean. However, since the population variance $S_{U}$ is always unknown, it needs to be replaced by its estimator $\hat{S}_{U}$ which, for a finite population, is given by [15]:

$\hat{S}_{U}=\frac{1}{n} \cdot \frac{N}{N-1} \cdot \sum_{k=1}^{n}\left(x_{k}-\hat{\bar{x}}_{U}\right)^{2}$

The variance of the estimator $\hat{S}_{U}$ depends on the selected sample (i.e. on the values $x_{k}$ of the $n$ elements observed) and is given by $[15]$ :

$$
\begin{aligned}
S\left(\hat{S}_{U}\right)= & \left(\frac{N}{N-1}\right)^{2} \cdot\left(\frac{1-(n / N)}{n}\right) \cdot \frac{1}{n-1} \\
& \cdot \sum_{k=1}^{n}\left[\left(x_{k}-\hat{\bar{x}}_{U}\right)^{2}-\frac{1}{n} \cdot \sum_{k=1}^{n}\left(x_{k}-\hat{\bar{x}}_{U}\right)^{2}\right]^{2}
\end{aligned}
$$

\section{Using finite population statistics to assess concrete strength in existing RC buildings}

\subsection{Discretizing the concrete strength and disaggregating its variability}

By depending on both $n$ and $N$, finite population statistics enable to control the epistemic uncertainty about the estimates of the mean and of the variability of a population using data provided by a ratio of $n / N$ elements. This approach is somehow similar to the uncertainty reduction principle that underlines the procedures in current standards (e.g. see [10]) where it is implicit that an increase in the number of structural elements that are tested during survey operations will lead to a reduction of the uncertainty about the estimate of the mean value of the material property. Therefore, a procedure based on finite population statistics like the one proposed herein is found to be consistent with current standard assessment procedures. 
Adopting finite population principles to assess the concrete strength in existing buildings requires additional considerations to define what can be considered to be a finite population of concrete strength values (i.e. a group of $N$ values where homogeneity is expected). To discretize the concrete strength values in a RC building and disaggregate them into finite populations, concrete strength variability was assumed to be the result of four components [3]: (1) within-test variability, (2) within-member variability, (3) between-member variability and (4) batch-to-batch variability. Systematic between-member (3) variability and batch-to-batch variability (4) were assumed to be dominant when compared with the other two components [3], despite their known effects (e.g. see $[9,14,16])$. This fact allows for the definition of a concrete strength discretization criterion where it is assumed that each structural member of the building is represented by a single concrete strength value. For the purpose of the proposed method, it is also considered that the concrete strength value of a given member can be assessed from a compression test performed on a concrete core extracted from the member. Using this discretization of the concrete strength values, the disaggregation of the (discrete) structure into finite populations of $N$ structural members can then be defined by analyzing the nature of the actual construction process of a building. It is noted that a similar rationale was adopted in [17] to disaggregate the concrete strength in existing RC buildings where the material properties were assumed to be homogeneous at each floor to reflect the construction timeline. Therefore, structural regions (where a region designates any cluster of structural elements that are believed to have similar physical properties, hence defining a finite population) can be established to reflect the expected batch-to-batch variability. These regions can be made by all the members of a given type in a storey (e.g. all the beams or all the columns), by a portion of the members of a given type in a storey or even by combining multiple types of elements in multiple storeys. A disaggregation following this strategy can be seen to be in agreement with the material property assessment procedures defined by current seismic safety assessment standards (e.g. see $[10,11,13])$. According to these procedures, the characterization of the concrete strength in a building must include data collected from each storey, from each type of structural element (e.g. columns, beams) and over an area with a limited size.

By disaggregating the concrete strength using this rationale, a number of regions can be defined within the building. Each one of these regions is a finite population with $N$ elements where concrete strength is expected to be homogeneous. Finite population statistics can then be used to assess the concrete strength in each region, namely by defining sampling plans in terms of the ratio $n / N$, i.e. the number of structural members of the region where the concrete strength is evaluated $(n)$ which is a fraction of the corresponding total number of structural members in the region $(N)$. Therefore, by defining statistics of the concrete strength (e.g. the mean value or other parameters) as a function of $n / N$, the level of epistemic uncertainty in the concrete strength assessment becomes explicitly controlled.

\subsection{Assessing the mean and the CoV of concrete strength using finite population statistics}

In order to see how finite population statistics can be applied to assess the mean value of the concrete strength, a simulation study is presented in the following where 8 datasets (referred hereon as $\mathrm{CH} 1-\mathrm{CH} 8$ ) were analyzed. Datasets $\mathrm{CH} 1-\mathrm{CH} 8$ have total sizes of $27,30,32,22,25,19,25$ and 27 and were extracted from [18] where further statistical details and analyses on these datasets can be found. The study presented herein replicates real conditions: an analyst must select a certain number of candidate structural elements $(n)$ of a region where the material strength will be assessed and no information about the remaining $(N-n)$ members will be available. For each dataset and for a given value of $n$, a number of samples were defined which correspond to the minimum between the number of possible combinations of $n$ elements extracted from the $N$ elements and 10 million random samples of size $n$ extracted from the $N$ elements. For each dataset, the lowest value of $n$ that was adopted was 2 and the largest was $N$.

In order to examine the sampling uncertainty about the mean estimate of the concrete strength due to the $(N-n)$ nonsurveyed structural elements, the ratio $\chi_{m}$ was defined:

$\chi_{m}=\frac{\hat{\bar{\chi}}_{U}}{\overline{\bar{x}}_{U}}$

where $\hat{\bar{x}}_{U}$ is the sample estimate of the mean (Eq. (4)) and $\bar{x}_{U}$ is the true population mean (Eq. (1)). Hence, for each size $n$, a dataset of $\chi_{m}$ values was created. In order to analyze how the sampling uncertainty about the mean is correlated with the global population variability, two parameters were analyzed: $\beta_{m, m}$ which is the mean of the $\chi_{m}$ ratios and $\beta_{\mathrm{Cov}, m}$ which is defined by:

$\beta_{\mathrm{CoV}, m}=\frac{\operatorname{CoV} \chi_{m}}{\operatorname{CoV}_{U}}$

where $\operatorname{CoV} \chi_{m}$ is the $\operatorname{CoV}$ of $\chi_{m}$ and $\mathrm{CoV}_{U}$ is the true population $\mathrm{CoV}$. Parameter $\beta_{\mathrm{CoV}, m}$ is closely related to the theoretical factor $\beta_{\mathrm{CoV}, m^{*}}$ which depends on the finite population correction factor and is defined by:

$\beta_{\mathrm{CoV}, m^{*}}=\frac{1}{\sqrt{n}} \cdot \sqrt{\frac{N-n}{N-1}}$

The results of the simulation study indicate that $\beta_{m, m}$ is 1.0 for all the considered sample sizes, which means that, on average, the population mean will be obtained from the samples, irrespectively of the adopted sample size (i.e. on average, $\chi_{m}=1$ ). With respect to the variability of $\chi_{m}$, Fig. 1 shows the evolution of $\beta_{\mathrm{CoV}, m}$ for increasing values of the ratio $n / N$ and for the datasets $\mathrm{CH} 1-\mathrm{CH} 8$ simultaneously, and compares it with the evolution of $\beta_{\mathrm{CoV}, m^{*}}$ calculated for different values of $N$ (from 10 to 40 in steps of 5).

As expected, the variability of $\chi_{m}$ decreases as the sample size increases. The rate of this reduction follows the evolution of $\beta_{\mathrm{CoV}, m^{*}}$ (evaluated for $N$ equal to the corresponding population size) and reduces $\beta_{\mathrm{Cov}, m}$ as $n / N$ converges to 1 . The value of $\beta_{\mathrm{Cov}, m}$ is also expected to vary significantly with the population size $N$, as can be seen from the $\beta_{\mathrm{Cov}, m^{*}}$ curves. It can be seen that, for a given value of $n / N, \beta_{\mathrm{Cov}, m^{*}}$ becomes lower as $N$ increases. This reduction is due to the fact that, for a given value of $n / N$, as $N$ increases, $n$ also

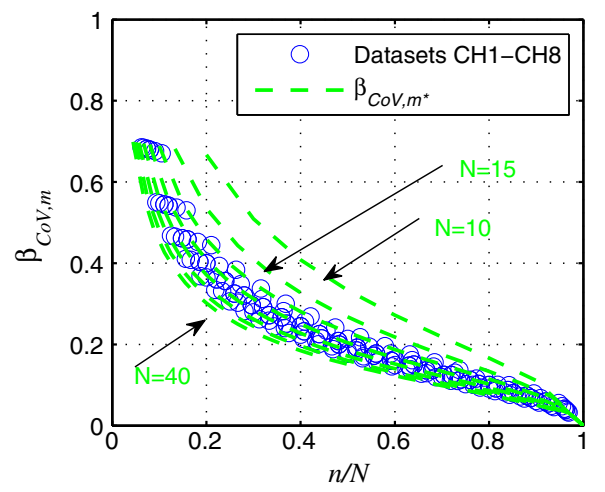

Fig. 1. Comparison between the $\beta_{\mathrm{Cov}, m}$ values obtained from the simulations (datasets $\mathrm{CH} 1-\mathrm{CH} 8$ ) and the values of $\beta_{\mathrm{CoV}, m^{*}}$ for different values of $N$ 
increases proportionally and the $1 / \sqrt{n}$ factor of Eq. (10) controls the $\beta_{\mathrm{Cov}, m^{*}}$ reduction rate.

Although, in the previous results, the estimate for the mean was analyzed assuming that $S_{U}$ (more precisely $\mathrm{CoV}_{U}$ ) was known (see Eq. (5)), often this value is not known and has to be estimated based on the sample results. Hence, it is also expected that statistical uncertainty will affect the estimate of $S_{U}$ obtained from a given sample due to the possibility of multiple combinations of $n$ out of $N$ test results (e.g. see Eq. (7)). The data that was simulated to analyze the ratio $\chi_{m}$ was therefore reused in order to examine the sampling uncertainty in the estimate of the population CoV, i.e. $\mathrm{CoV}_{U}$. Parameter $\mathrm{CoV}_{U}$ was selected as a measure of the population dispersion instead of the variance $S_{U}$ since it quantifies the variability without scaling effects, i.e. without depending on the range of values of the population. This new analysis examined the ratio $\chi_{\mathrm{CoV}}$ defined by:

$\chi_{\mathrm{CoV}}=\frac{\hat{\mathrm{CoV}}_{U}}{\mathrm{CoV}_{U}}$

where $\hat{C}_{0} V_{U}$ is the $\mathrm{CoV}$ estimated using the sampled data and $\operatorname{CoV}_{U}$ is the corresponding true population value. The mean and the $\mathrm{CoV}$ of $\chi_{\mathrm{Cov}}$ were analyzed for different values of $n / N$ to verify the rate at which $\hat{C}_{0} V_{U}$ converges to the real value $\operatorname{CoV}_{U}$ in typical populations of concrete core strength values. Fig. 2a presents the evolution of the mean of $\chi_{\mathrm{CoV}}$ and Fig. $2 \mathrm{~b}$ presents the evolution of the CoV of $\chi_{\mathrm{Cov}}$ for increasing values of $n / N$. In both cases, analytical approximations were fitted to evaluate the evolution of the mean and of the $\mathrm{CoV}$ of $\chi_{\mathrm{CoV}}$ as a function of $n / N$.

From Fig. 2a, it can be seen that only the results of dataset $\mathrm{CH} 1$ are not in close agreement with the trend line established for the mean of $\chi_{\text {cov }}$ which is defined by the power model given by:

$$
\text { Mean } \chi_{\mathrm{CoV}}=1.01-0.01 \cdot(n / N)^{-1.16}
$$

As can be seen in Fig. 2a, a mean ratio of 0.95 is obtained for $n / N$ equal to 0.2 , while for the $\mathrm{CH} 1$ dataset a mean ratio of 0.95 requires an increase of $n / N$ up to 0.35 . For the variability of $\chi_{\mathrm{Cov}}$, its power decay with the increase of $n / N$ can be defined by:

$$
\operatorname{CoV} \chi_{\mathrm{CoV}}=0.22 \cdot(n / N)^{-0.56}-0.16
$$

For this case, the trend line that was found is consistent with all the datasets. As can be seen from Fig. 2b, the uncertainty about the estimate of the population $\mathrm{CoV}$ requires higher sample sizes in order to achieve acceptable levels of precision. For example, at least $40 \%$ of the total number of structural elements have to be tested in order to get a minimum $\operatorname{CoV} \chi_{\mathrm{CoV}}$ of 0.20 .

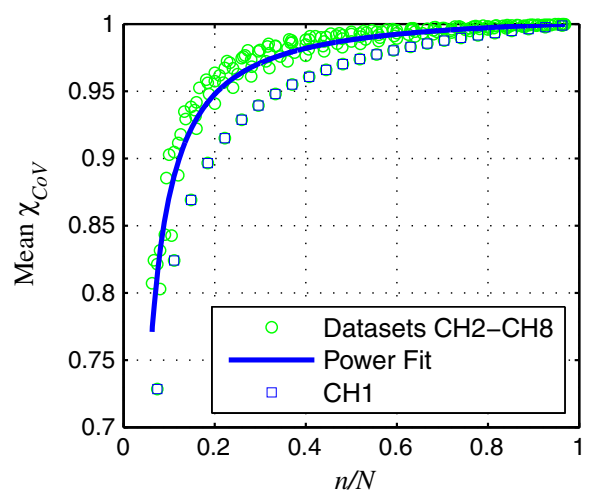

(a)
To further illustrate the impact of sampling in the assessment of the CoV, Fig. 3 shows the boxplots of $\chi_{\mathrm{Cov}}$, obtained for all the datasets when adopting ratios of $3 / N$ (Fig. $3 a$ ) and $6 / N$ (Fig. $3 b$ ). These two ratios were selected because they correspond to the minimum sample sizes proposed in [11].

As shown in Fig. 3, the distribution of the sampling CoV (in this case represented by the ratio $\chi_{\text {cov }}$ ) is considerably asymmetric. Furthermore, this visual asymmetry is seen to reduce when the sample size increases from $n=3$ (Fig. 3a) to $n=6$ (Fig. 3b). Based on these results, it becomes clear that taking a small sample of values from a population of concrete cores may lead to a significant overestimation or underestimation of the concrete strength variability.

In the overall, the simulation study results indicate that adopting the proposed finite structure paradigm and using finite population principles to assess concrete strength statistics provides important information regarding the reduction of uncertainty when increasing the ratio $n / N$. Furthermore, the results also show that a high (and often impractical) number of destructive tests is required to reduce the epistemic uncertainty to acceptable levels (i.e. for $\mathrm{CoV} \chi_{\mathrm{CoV}}$ to be around 0.10). Therefore, alternative methods must be defined to estimate the concrete strength variability in a finite population and overcome the need to carry out a high number of destructive tests.

\section{An alternative method to estimate the finite population CoV of concrete strength}

An alternative approach is proposed herein to estimate the variability (i.e. the $\mathrm{CoV}$ ) of a finite population of concrete strength values using auxiliary information obtained from non-destructive tests (NDTs). These tests are often used in survey campaigns since they induce limited levels of damage to the structural components and can be used in a larger number of elements usually at a lower cost. An example of this kind of methods is the surface hardness determination test using the rebound hammer. The results of this test have been shown to correlate well with the concrete compressive strength and multiple correlation models have already been proposed (e.g. see [19]). When using adequately calibrated correlation models, the measured rebound numbers (RNs) can be converted into compressive strength estimates. Still, it is noted that current standards (e.g. see [20]) do not allow the use of these correlations without a preliminary calibration involving destructive tests results (at least 9) obtained from concrete cores collected from the building under survey. In general, standard-based methods recommend the use of NDTs as a complementary source of information to assess existing structures. As an example, Masi and Vona [6] recommended conducting NDTs in $8 \%$ to $15 \%$ of the total number

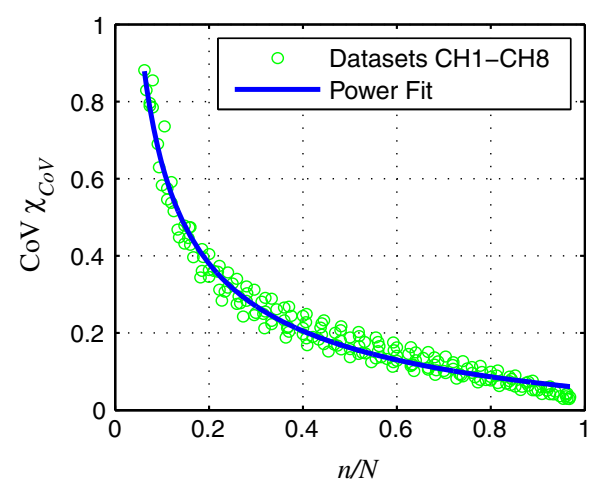

(b)

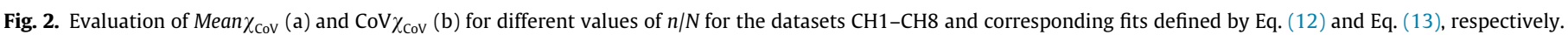



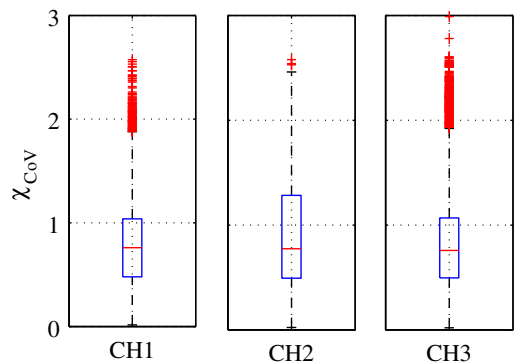

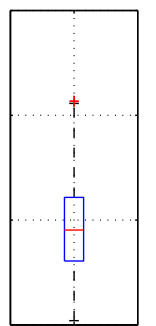

$\mathrm{CH} 4$

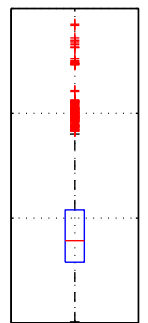

CH5

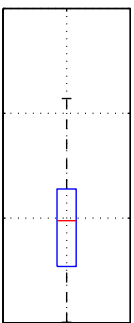

CH6

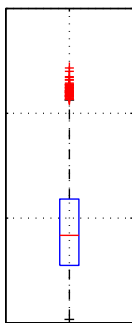

$\mathrm{CH} 7$

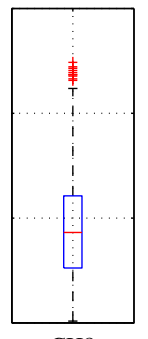

CH8

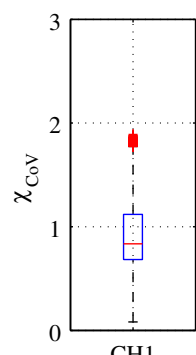

$\mathrm{CH} 1$

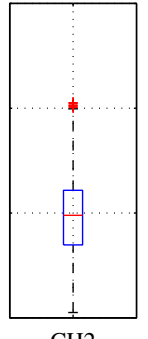

$\mathrm{CH} 2$

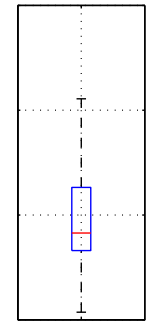

$\mathrm{CH} 3$

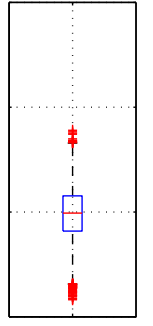

$\mathrm{CH} 4$

(a)

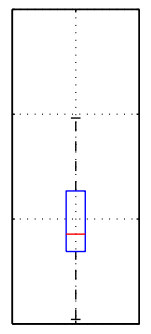

CH5

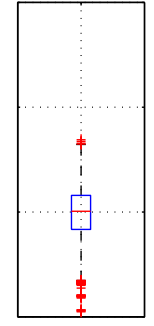

CH6

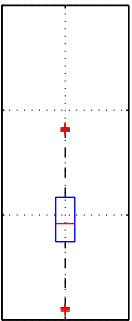

$\mathrm{CH} 7$

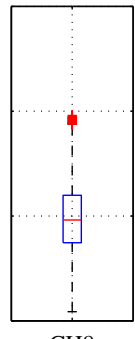

CH8

(b)

Fig. 3. Boxplots of the $\chi_{\mathrm{CoV}}$ ratios obtained when sampling from $\mathrm{CH} 1-\mathrm{CH} 8$ with sizes (a) $n=3$ and (b) $n=6$.

of elements per storey, with an absolute minimum of 6 to 10 tests. For example, in a region that has 20 structural elements, this leads to a minimum number of tests corresponding to $n / N=0.30$.

Instead of converting the $\mathrm{RN}$ test results into concrete strength values, the proposed method defines a direct correlation between the $\mathrm{CoV}$ of destructive tests $\left(\mathrm{CoV}_{f c}\right)$ and the $\mathrm{CoV}$ of the RNs $\left(\mathrm{CoV}_{R N}\right)$ evaluated for the same structural elements. Pairs of data comprising CoVs of populations of RNs and concrete core compressive test results were selected from existing literature studies [21-23] to establish the proposed model. A total of $24 \mathrm{CoV}_{f c}-\mathrm{CoV}_{R N}$ pairs were used, each one comprising more than 8 locations/readings of both tests. Fig. 4 shows the correlation obtained for the considered data, together with the $75 \%$ prediction bounds of the model [24]. The correlation was derived using a robust regression model with a bi-squared weighting function. The global correlation model that was obtained has an adjusted- $R^{2}$ of 0.72 , a root mean squared error (RMSE) of 0.06 and is expressed by:

$\hat{\mathrm{CoV}}_{f \mathrm{c}}=1.042 \cdot \mathrm{CoV}_{R N}+0.123$

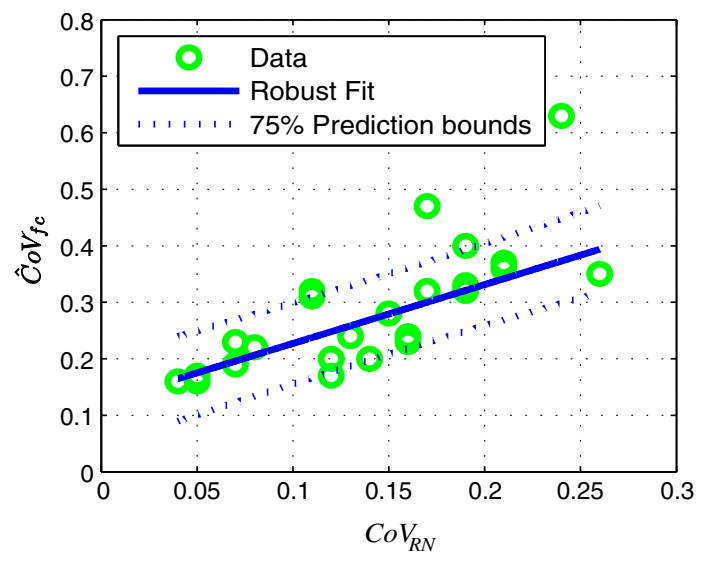

Fig. 4. Correlation between $\hat{C}_{0} V_{f c}$ and $\operatorname{CoV}_{R N}$ along with the corresponding $75 \%$ prediction bounds.
The model requires the variability of the RN values to be known, i.e. an adequate estimate must be defined for $\operatorname{CoV}_{R N}$ and the indicative sample sizes proposed in [6] can be used as a reference to establish this estimate. Accordingly, for the ranges proposed (610 tests per storey, which, under the finite population paradigm, means per finite population), it is assumed that values of $n / N$ in the range 0.30-0.40 will yield acceptable estimates of $C o V_{R N}$.

The purpose of defining this general model correlating the variability of both tests was to check if it was possible to derive a tool that would, without any calibration, provide an indication of the variability that an analyst may expect prior to the design of the destructive test campaign. Hence, a naïve approximation for the $\operatorname{CoV}_{f c}$ was analyzed to check what would be the possible improvements on the quality of the estimation of the variability or the mean when compared with other strategies. It must be noticed that the developed approximation [Eq. (14)] was used hereon as a benchmark due to the limited amount of datasets available in literature. The data used to construct was extracted from populations of data from experimental campaigns performed in a laboratory or in situ, using possibly multiple types of equipment and different operation qualities. Hence, the proposed law can be seen as a general methodology and future improvements using results from experimental campaigns performed locally at each country in portfolios of existing buildings may significantly improve the robustness and reduce the generality of the benchmark adopted herein.

Finally, by assuming that $\hat{C} o V_{f c}$ provides an adequate estimate of $\mathrm{CoV}_{U}$ and considering that $\beta_{\mathrm{Cov}, m^{*}}$ represents the theoretical evolution of $\beta_{\mathrm{CoV}, m}$ (Fig. 1), a reliable estimate of the sampling variability of the mean estimate for the concrete strength $\operatorname{CoV}\left(\hat{\bar{x}}_{f c}\right)$ in typical storeys (i.e. with $N$ structural elements in the range of 15-30) is obtained by combining Eq. (14) and Eq. (10):

$$
\begin{gathered}
\beta_{\operatorname{CoV}, m}=\frac{\operatorname{CoV} \gamma_{m}}{\operatorname{CoV}_{U}}=\frac{\operatorname{CoV}\left(\hat{x}_{f c}\right)}{\operatorname{CoV}_{U}} \Longleftrightarrow \\
\Longleftrightarrow \operatorname{CoV}\left(\hat{\bar{x}}_{f c}\right)=\beta_{\operatorname{CoV}, m^{*}} \cdot \operatorname{CoV}_{U} \Longleftrightarrow \\
\Longleftrightarrow \operatorname{CoV}\left(\hat{\bar{x}}_{f c}\right)=\left(\frac{1}{\sqrt{n}} \cdot \sqrt{\frac{N-n}{N-1}}\right) \cdot\left(1.042 \cdot \operatorname{CoV}_{R N}+0.123\right)
\end{gathered}
$$


where $C o V_{R N}$ needs to be determined from a minimum of $n / N=0.30$ tests.

\section{Validation of the proposed procedure using experimental data}

To assess the validity of the proposed finite population approximations defined by Eqs. (14) and (15), five additional datasets of RN and core strength values were considered. Datasets C1-C4 correspond to pairs of data extracted from multi-storey RC buildings constructed in the mid-1990 s that were surveyed within the present study. Each pair has a core strength value evaluated in a structural element and a RN value from the same location. Since dataset C4 presented a wide range of concrete strength values (from 20.75 MPa to $64.81 \mathrm{MPa}$ ) a subset of C4 (termed C4*) was additionally defined where the top five values were removed in order to obtain a more homogeneous dataset. Dataset C5 was obtained from [25] and comprises RN and concrete core strength values extracted from an existing building. Table 1 summarizes the selected datasets.

A simulation study was performed to evaluate the reliability of the proposed correlation defined by Eq. (14) to estimate the variability of the concrete strength $\hat{C} o V_{f c}$ by analyzing the empirical cumulative distribution function (ECDF) of the ratio $\psi_{\mathrm{Cov}}$ defined by:

$\psi_{\mathrm{CoV}}=\frac{\hat{\operatorname{Co}} V_{f c}}{\operatorname{CoV}_{f c}}$

Where $\hat{C} o V_{f c}$ represents the estimate of the real variability $\operatorname{CoV}_{f c}$ obtained for each dataset when assessing $n$ out of $N$ randomly selected structural elements without having information on the remaining $N-n$ structural elements. To estimate $\hat{C}_{o} V_{f c}$ for each dataset (C1-C5), M samples (see Eq. (3)) with sizes $n / N=0.30$ were extracted (i.e. $n$ equal to $6,8,6,8,6$, and 6 tests for datasets $\mathrm{C} 1-\mathrm{C} 5$, respectively). For each sample, the value of $\operatorname{CoV}_{R N}$ was converted into the estimate $\hat{C} o V_{f c}$ using two different models: Model RMP1 which corresponds to the correlation defined by Eq. (14), and model RMP2 which is a variant of this model that considers a $50 \%$ upper confidence bound of the regression, assuming the normality of the residuals and adding $0.6745^{*}$ RMSE to the mean prediction, and is given by:

$\hat{C}_{0} V_{f c}=1.042 \cdot \operatorname{CoV}_{R N}+0.163$

Based on this regression model, a rationale similar to the one leading to Eq. (15) can also be established to define a new estimate for $\operatorname{CoV}\left(\hat{\bar{x}}_{f c}\right)$ now given by:

$\operatorname{CoV}\left(\hat{\bar{x}}_{f c}\right)=\left(\frac{1}{\sqrt{n}} \cdot \sqrt{\frac{N-n}{N-1}}\right) \cdot\left(1.042 \cdot \operatorname{CoV}_{R N}+0.163\right)$

In order to compare the uncertainty associated to these strategies with others that involve the use of correlation models converting each value of RN into a point estimate for the concrete strength $f_{c}$, the simulated results of $\psi_{\mathrm{Cov}}$ for the RMP1 and RMP2 models

Table 1

Statistical parameters of the six datasets considered in the validation study.

\begin{tabular}{lllll}
\hline Dataset & $N$ & $\bar{x}_{U}(\mathrm{MPa})$ & $\mathrm{CoV}_{f c}$ & $\mathrm{CoV}_{R N}$ \\
\hline C1 & 19 & 27.46 & 0.29 & 0.17 \\
$\mathrm{C} 2$ & 27 & 28.11 & 0.36 & 0.21 \\
C3 & 20 & 30.14 & 0.38 & 0.16 \\
C4 & 25 & 35.99 & 0.34 & 0.14 \\
C4 & 20 & 30.66 & 0.18 & 0.12 \\
C5 & 21 & 19.74 & 0.19 & 0.08 \\
\hline
\end{tabular}

were reused for a secondary analysis. In this case, the objective was to derive the ECDF of the $\psi_{\mathrm{Cov}}$ ratios after converting the $n$ RN values of the $M$ samples into $f_{c}$ values using correlation models (RM) from the literature. After converting the RN values, statistical analyses of each sample were performed and $M$ possible estimates of $\hat{C} o V_{f c}$ were computed. Again, the $\psi_{\mathrm{cov}}$ values were calculated by normalizing the $M \hat{C}_{o} V_{f c}$ values by the $\operatorname{CoV}_{f c}$ of the corresponding dataset. Three different RM models were considered to convert the RN values into $f_{c}$ values. The RM1 and RM3 correlation models were selected because they were derived using data that is believed to be similar to the datasets $\mathrm{C} 1-\mathrm{C} 5$ considered herein. The correlation model RM2 was selected due to its alternative form. Model RM1 is a power model proposed in [26] and defined by:

$f_{c, \mathrm{RM} 1}=0.00917 \cdot(\mathrm{RN})^{2.27}$

Model RM2 is the calibration curve proposed in [27] assuming $f_{c, r e f}=30$ and $\mathrm{RN}_{r e f}=35$, given by:

$f_{c, \mathrm{RM} 2}=f_{c, r e f} \cdot\left(\frac{\mathrm{RN}}{\mathrm{RN}_{r e f}}\right)^{2.38}$

The model termed RM3 is the power model fitted to the dataset C5 in [25] and defined by:

$f_{c, \mathrm{RM} 3}=0.00645 \cdot(\mathrm{RN})^{2.23}$

Although the proposed procedure focusses on quantifying $\hat{C} o V_{f c}$, the analysis of the RM models also allows for the computation of an estimate for the mean concrete strength of each dataset, $\hat{\bar{x}}_{f c}$. Due to the importance of having an estimate for this statistical parameter, the reliability of RM1, RM2 and RM3 was also evaluated with respect to $\hat{\bar{x}}_{f c}$. This additional analysis only requires computing the mean of the $n$ converted values of $f_{c}$ for each one of the $\mathrm{M}$ samples. To evaluate the statistical uncertainty associated with $\hat{\bar{x}}_{f c}$, the ECDF of the M ratios $\psi_{m}$ was analyzed, where $\psi_{m}$ is given by:

$\psi_{m}=\frac{\hat{\bar{x}}_{f c}}{\bar{x}_{f c}}$

in which $\bar{x}_{f c}$ is the mean of the concrete strength of the corresponding dataset ( $\mathrm{C} 1-\mathrm{C} 5)$.

It is noted that the objective of using the selected correlation models was to verify how the estimates of $\psi_{m}$ and $\psi_{\mathrm{cov}}$ would compare in terms of sampling uncertainty with that of core samples with sizes $n=3$ and $n=6$. These sample sizes were selected as benchmarks because standards often refer them as values for the minimum number of tests that need to be carried out under several situations (e.g. see [10-13]). Furthermore, for the selected datasets, $n=3$ corresponds to an average value of $n / N$ equal to 0.14 while $n=6$ corresponds to an average value of $n / N$ equal to 0.28 . The $\mathrm{M}$ combinations of destructive test results (i.e. core strength values $f_{c}$ ) were extracted from datasets $\mathrm{C} 1-\mathrm{C} 5$ and for each one of the $\mathrm{M}$ samples, the estimates of $\hat{C}_{o} V_{f c}, \hat{\bar{x}}_{f c}, \psi_{m}$ and $\psi_{\text {cov }}$ were computed. In addition, the ECDF of the $\mathrm{M} \psi_{m}$ and $\psi_{\mathrm{Cov}}$ ratios was computed for each dataset to compare them with those calculated based on RMP1, RMP2, RM1, RM2 and RM3.

Finally, the efficiency of Eqs. (15) and (18) to estimate the real sampling uncertainty of the mean was also analyzed. The values of $\hat{C} o V_{f c}$ were used as an input in Eqs. (15) and (18) to estimate $\operatorname{CoV}\left(\hat{\bar{x}}_{f c}\right)$. This analysis involved four models: $\operatorname{SIM}_{n=3}$ which involve the estimate of $\operatorname{CoV}\left(\hat{\bar{x}}_{f c}\right)$ obtained using Eq. (15) (RMP1) and $n=3$, $\operatorname{SIM}_{n=3}^{*}$ which involve the estimate of $\operatorname{CoV}\left(\hat{\bar{x}}_{f c}\right)$ obtained with Eq. (18) (RMP2) and $n=3, \operatorname{SIM}_{n=6}$ which involve the estimate of $\operatorname{CoV}\left(\hat{\bar{x}}_{f c}\right)$ obtained with Eq. (15) (RMP1) and $n=6$ and $\operatorname{SIM}_{n=6}^{*}$ which 
involve the estimate of $\operatorname{CoV}\left(\hat{\bar{x}}_{f c}\right)$ obtained with Eq. (18) (RMP2) and $n=6$. The ECDF of the $\mathrm{M} \operatorname{CoV}\left(\hat{\bar{x}}_{f c}\right)$ values that result from the M possible estimates of $\hat{C} o V_{f c}$ was then calculated for all these models. To analyze the performance of Eq. (15), the EDCF curves were compared with the $\operatorname{CoV}\left(\hat{\bar{x}}_{f c}\right)$ (a scalar value) obtained when computing the $\mathrm{CoV}$ of all the $\mathrm{M}$ estimates of $\hat{\bar{x}}_{f c}$ when using 3 destructive tests $\left(\operatorname{Real}_{n=3}\right)$ and 6 destructive tests ( Real $\left._{n=6}\right)$.

\section{Results and discussion}

\subsection{Analysis of the $\psi_{\mathrm{Cov}}$ ratios}

Fig. 5 presents the ECDFs of the $\psi_{\mathrm{Cov}}$ ratios obtained using the different strategies defined in the previous Section (i.e. RMP1, RMP2, RM1, RM2 and RM3). As mentioned before, all the computed ECDFs are conditioned to a sample size corresponding to $n$ / $N=0.30$. Hence, the presented ECDFs reflect the sampling uncertainty associated with the selection of different test locations for the rebound hammer test within a given finite population.

The results show that the RMP1 and RMP2 models lead to data with a lower dispersion when compared with that obtained from models RM1, RM2 and RM3. This trend can be observed by analyzing the steepness of the ECDFs, which is higher for the RMP1 and RMP2 models than for the other cases. The median estimate obtained for $\psi_{\mathrm{CoV}}$ with RMP1 changes with the considered dataset. In some cases it is higher than 1.0 (Fig. 5e) while in others it is lower than 1.0 (Fig. 5c and d). In the overall, the RMP2 model provided results that are more conservative than the RMP1 model which underestimated the median ratio for datasets C3 and C4 (Fig. 5c and d). Regarding model RM3, it should be noted that the sampling uncertainty has a significant effect in the estimation of the CoV even for dataset C5 (the dataset for which the RM3 model was calibrated), (Fig. 5f). From Fig. 5, it can also be seen that models RM1 and RM2 lead to data with a dispersion similar to that of
RM3, possibly due to the closeness of the exponents of the power term (see Eqs. (19)-(21)).

It is noted, however, that the models RM1, RM2 and RM3 that were selected are not representative of all possible models. To demonstrate the impact of selecting different regression models (i.e. with different values of the fitted parameters) in the estimate of $\psi_{\mathrm{Cov}}$, an additional analysis was performed using artificial power models (RMb) $a x^{b}$ simulating different values of the fitted parameters. Power models with an exponent term $b$ between 1.0 and 3.0 in steps of 0.25 were simulated. The term $a$ was estimated for each value of $b$ using the correlation between the coefficients derived by the meta-analysis presented in [28] (i.e. $b=1.0307-0.259 \cdot \ln$ (a)). Fig. 6 shows the ECDFs of the $\psi_{\mathrm{Cov}}$ that were obtained for datasets $\mathrm{C} 1, \mathrm{C} 3$ and $\mathrm{C} 5$ using the several RMb correlation models. For comparison purposes, the ECDFs of the $\psi_{\mathrm{Cov}}$ that were obtained with RMP1 and RMP2 are also shown. These results indicate that a lower dispersion of $\psi_{\mathrm{CoV}}$ can be obtained when selecting a value $b$ equal to $1.75,2.25$ and 3.0 for datasets $C 1, C 3$ and $C 5$, respectively. From these results, it can be seen that estimating the variability of the concrete strength using predefined correlation models (i.e. with uncalibrated values of $a$ and $b$ ) can lead to large and unreliable values. Furthermore, it is seen that in some cases (Fig. 6c) none of the RMb models is able to capture the correct trend (which is approximately captured by the models RMP1 and RMP2). This shows that, even when using calibrated correlation models (assuming that the referred correlation between the parameters $a$ and $b$ is a valid approximation), caution must be adopted when estimating the $\mathrm{CoV}$ of the concrete strength.

Fig. 7 presents the comparison of the ECDFs of the $\psi_{\mathrm{Cov}}$ ratios obtained using the RMP1 and RMP2 models and using all the possible samples with $n=3$ and $n=6$ cores of each dataset.

The observations that can be made regarding these results are twofold. Firstly, it can be seen that blindly selecting a small sample of cores within a finite population (an approach that is in agreement with current standards) may lead to inadequate estimates of the variability since the dispersion exhibited by the corresponding ECDF

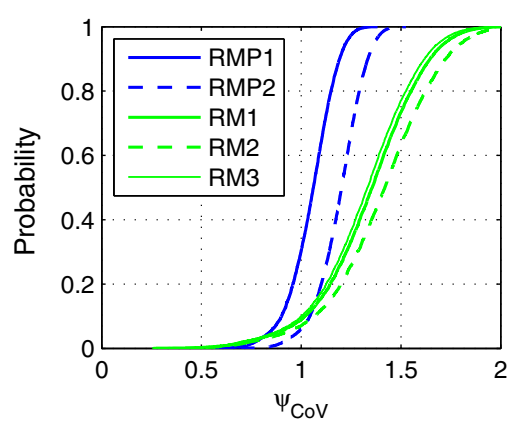

(a)

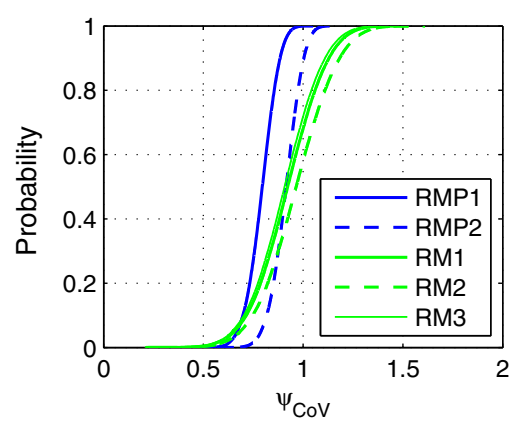

(d)

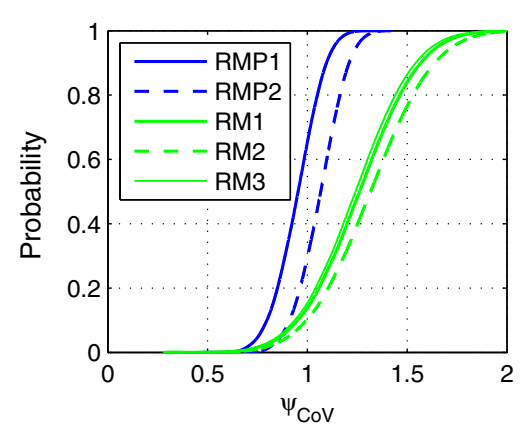

(b)

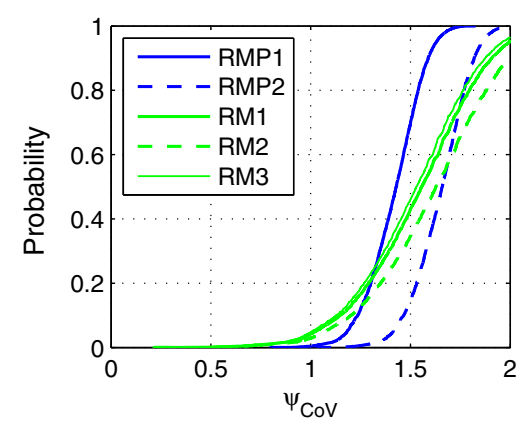

(e)

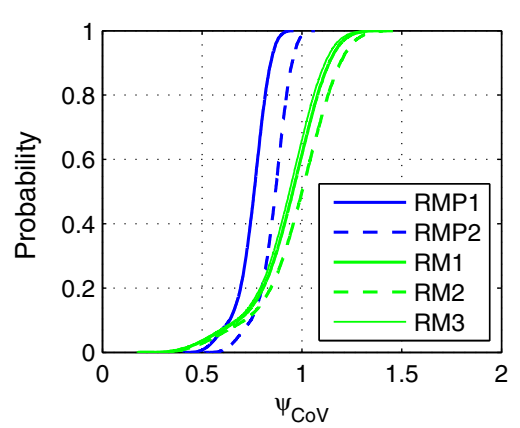

(c)

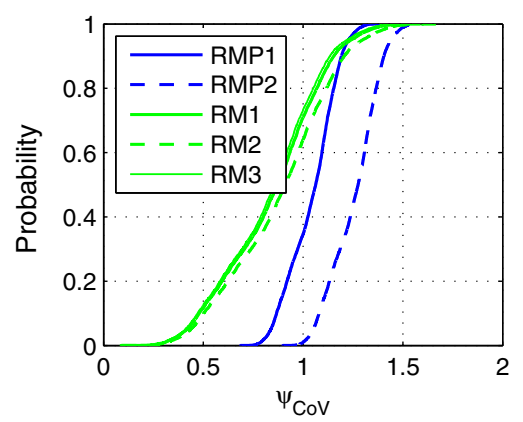

(f)

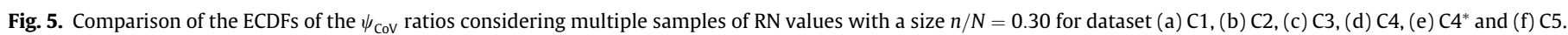




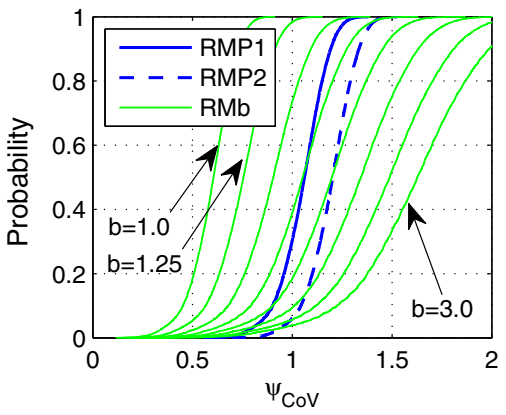

(a)

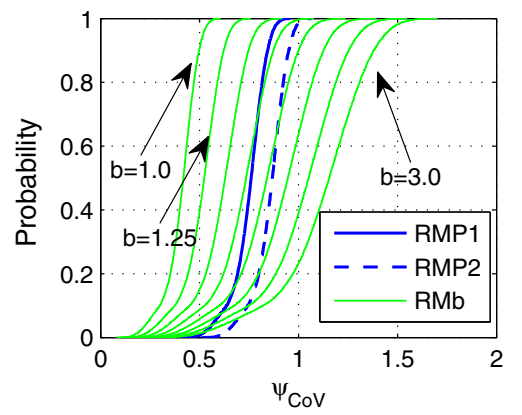

(b)

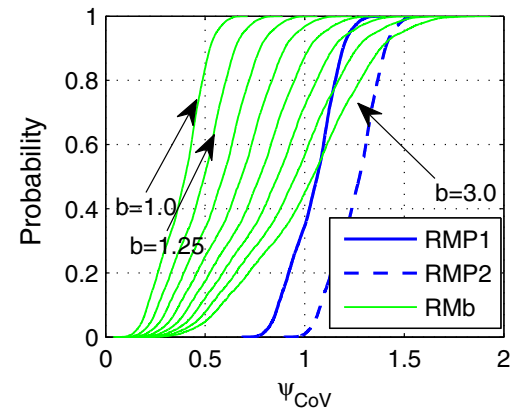

(c)

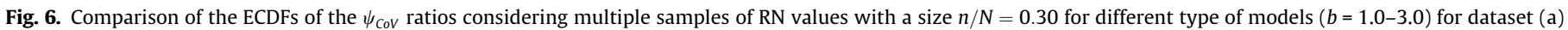
C1, (b) C3 and (c) C5.

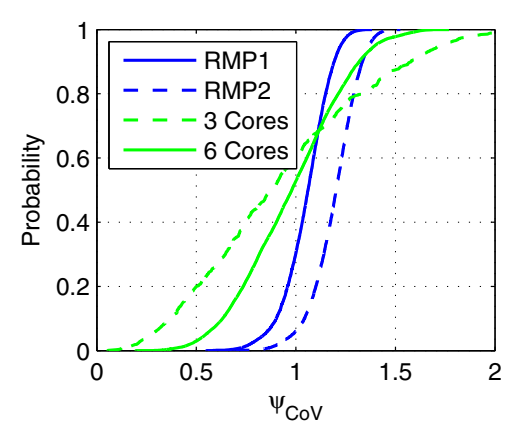

(a)

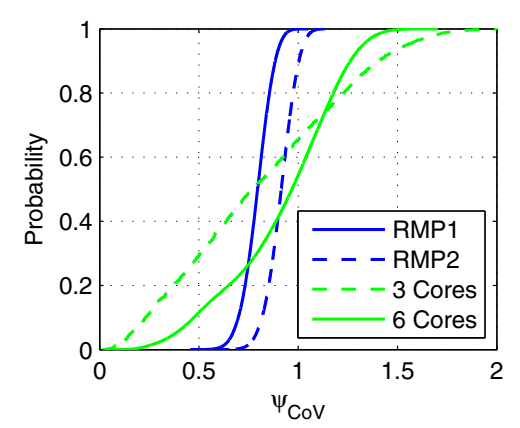

(d)

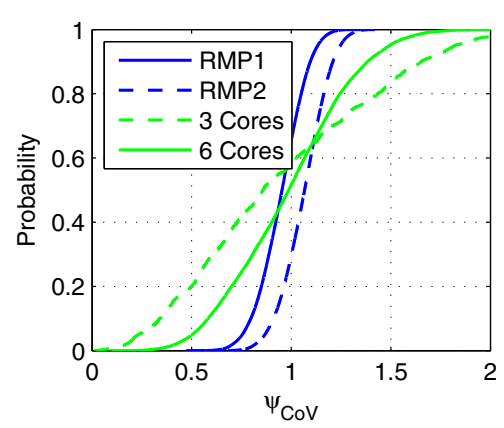

(b)

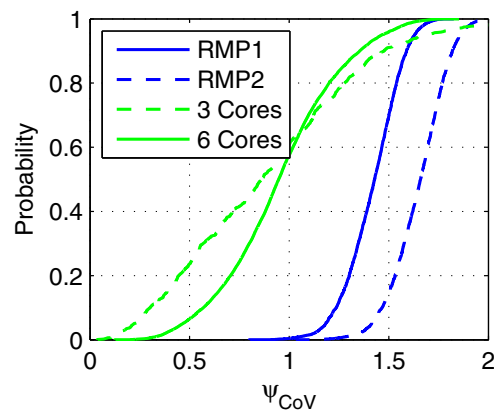

(e)

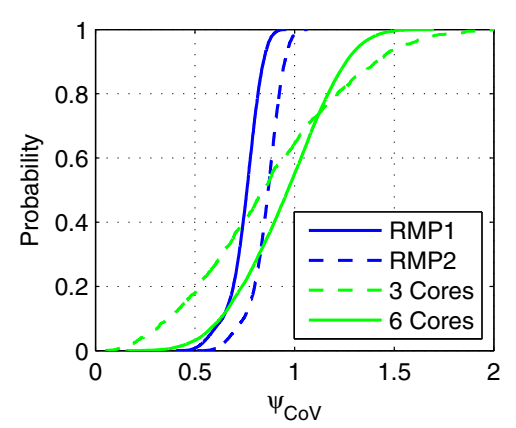

(c)

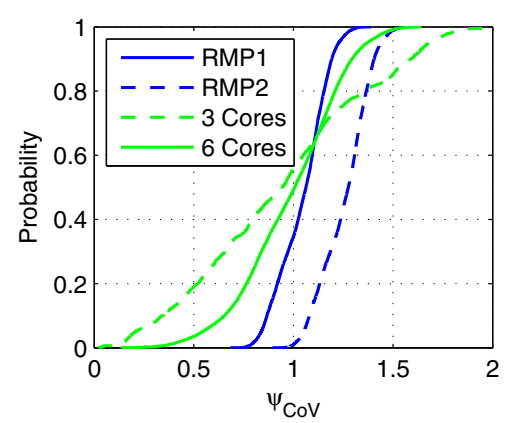

(f)

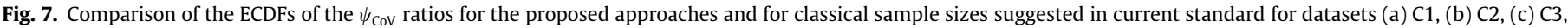
(d) $\mathrm{C} 4$, (e) $\mathrm{C} 4^{*}$ and (f) $\mathrm{C} 5$.

curves is very large. These observations are consistent across all the datasets, irrespective of the fact that they might have a higher (e.g. C1-C4) or a lower (e.g. C5) dispersion.

Secondly, when comparing these results with the proposed strategies (RMP1 and RMP2), it can be seen that the statistical uncertainty is adequately managed when using the RN values to estimate the variability. This confirms the suggestion in [14] that highlights the potential use of NDTs to complement the use of core strength values to assess the concrete strength in existing buildings. The results presented herein are not only in agreement with [14] but also show that the proposed methodology improves the previous use of NDTs since it reduces the uncertainty in the estimation of the concrete strength variability.

To further highlight this conclusion, Fig. 8 shows a global parametric comparison between the results obtained by the RMP1, RMP2, RM1, RM2 and RM3 models and the core-based strategies with $n=3$ and $n=6$. This comparison is performed for the Mean $\psi_{\mathrm{CoV}}$ (Fig. 8a) and the $\mathrm{CoV} \psi_{\mathrm{CoV}}$ (Fig. 8b).
The analysis of these parameters indicates that the RMP1 and RMP2 models provide adequate results, especially in terms of controlling the uncertainty given by $\mathrm{CoV} \psi_{\mathrm{Cov}}$ (Fig. 8b). With respect to the Mean $\psi_{\mathrm{CoV}}$, RMP1 underestimates the expected value of the population $\mathrm{CoV}$ (the range of Mean $\psi_{\mathrm{Cov}}$ is $0.75-1.05$ ) while RMP2 provides more conservative results (the range of Mean $\psi_{\mathrm{Cov}}$ is $0.86-1.26$ ). The best average response was observed when using the $n=6$ cores approach (the range of Mean $\psi_{\mathrm{Cov}}$ is $0.92-$ 0.99). Nevertheless, if, on average, an adequate estimate of the population $\mathrm{CoV}$ can be obtained when using the strength results of 6 cores, analyzing the expected variability (Fig. 8b) shows otherwise. The values of $\mathrm{CoV} \psi_{\mathrm{cov}}$ for this approach range from 0.25 to 0.31 which indicates that taking a random sample of size 6 from all the possible structural elements may yield significantly variable estimates of the population $\mathrm{CoV}$. Still, the worst results in terms of $\mathrm{CoV} \psi_{\mathrm{Cov}}$ are observed when samples with $n=3$ cores are considered since the range of $\mathrm{CoV} \psi_{\mathrm{CoV}}$ is now $0.45-0.54$. On the contrary, the most precise estimates of $\psi_{\mathrm{Cov}}$ are given by the RMP1 and 


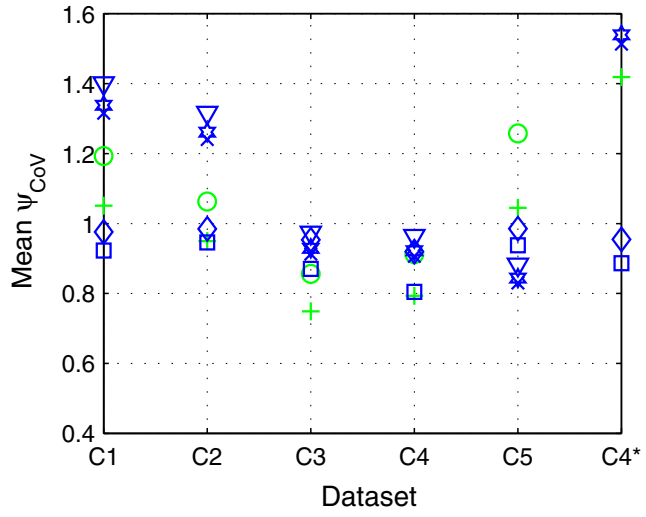

(a)

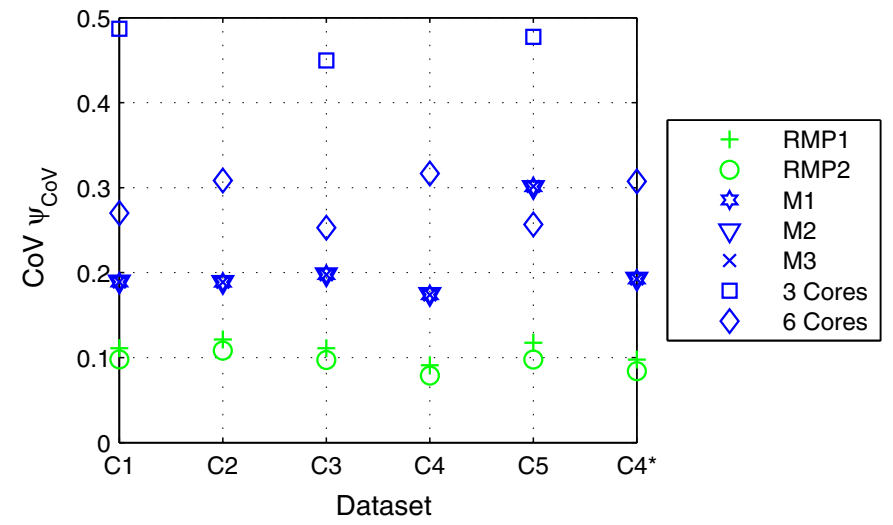

(b)

Fig. 8. Statistical analysis of $\psi_{\mathrm{CoV}}$ for different models and datasets: (a) analysis of the Mean $\psi_{\mathrm{CoV}}$, (b) analysis of the CoV $\psi_{\mathrm{Cov}}$.

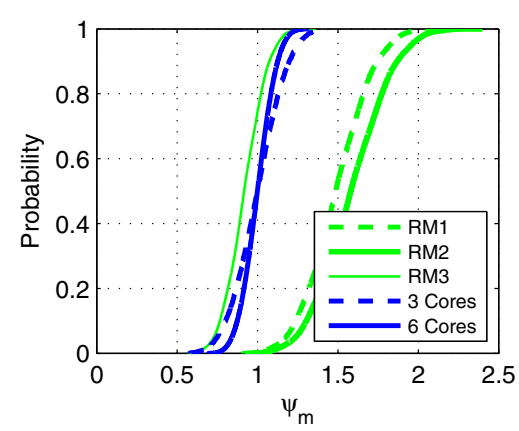

(a)

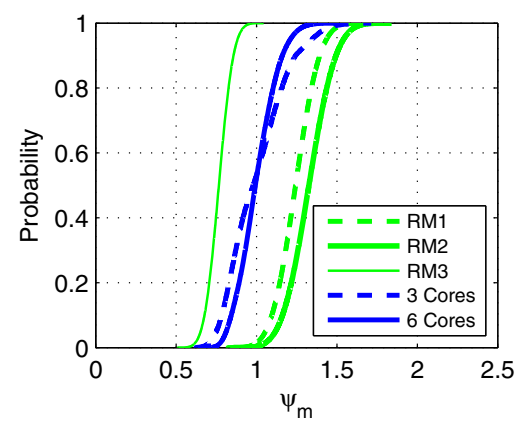

(d)

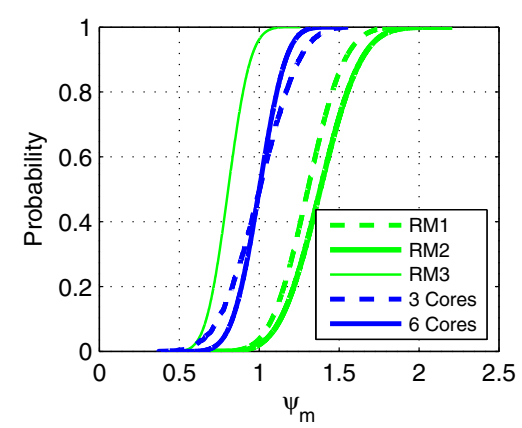

(b)

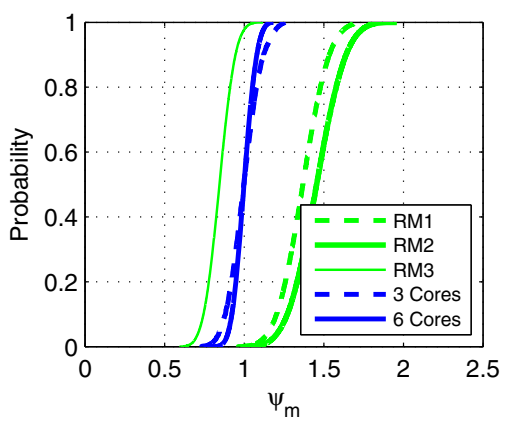

(e)

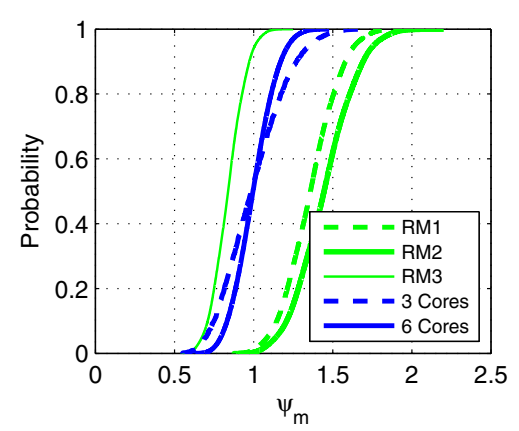

(c)

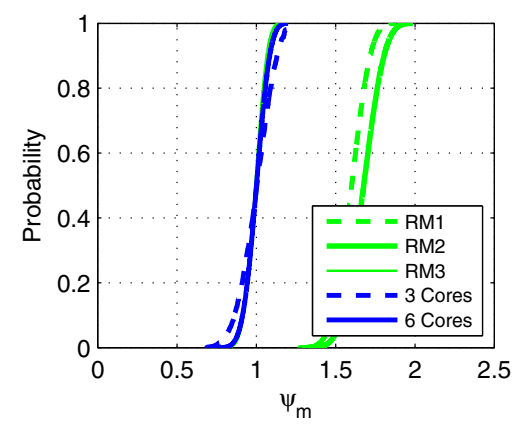

(f)

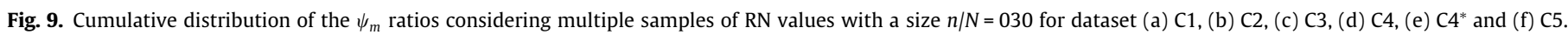

RMP2 models, which exhibit values of $\operatorname{CoV} \psi_{\mathrm{Cov}}$ that range from 0.09 to 0.11 and from 0.08 to 0.11 , respectively.

\subsection{Analysis of the $\psi_{m}$ ratios}

With respect to the results of the $\psi_{m}$ ratios, Fig. 9 presents the ECDFs of the estimates obtained using the samples of RN values and the models RM1, RM2 and RM3. These curves are compared with those obtained with the core-based strategies with $n=3$ and $n=6$ in order to verify if the use of predefined correlation models would lead to a lower sampling variability when compared to that which is obtained using with core samples with sizes $n=3$ and $n=6$. It can be seen that, on average, the RM1, RM2 and RM3 models fail to predict the true mean of the population since the median value of the ECDFs is, in most cases, shifted away from the ratio $\psi_{m}=1$. An exception is however found for dataset C5 and model RM3 (Fig. 9f) since this model was calibrated for this dataset. Despite this agreement of results when estimating the mean of the concrete strength, it is recalled that this model was unable to provide adequate estimates of the $\mathrm{CoV}$ of this dataset (Fig. 5f). With respect to the core-based strategies with $n=3$ and $n=6$, they can be seen to provide adequate estimates for the mean of the finite population. Furthermore, no significant differences have been found between the results for $n=3$ or $n=6$ cores.

Finally, Fig. 10 shows the comparison between the values of $\operatorname{CoV}\left(\hat{\bar{x}}_{f c}\right)$ obtained with the core-based strategies with $n=3$ and $n=6$ and the corresponding ECDF curves involving different samples of RN values, i.e. RMP1 (Eq. (15)) and RMP2 (Eq. (18)). The results show that, for all the cases and models considered, the ECDFs have a small variability and the difference between their median value and the CoV of the sample mean is usually within a range of 0.05 , thus demonstrating the adequacy of the proposed approaches. The differences found are a direct consequence of the main limitation of the proposed methods, i.e. they rely on 


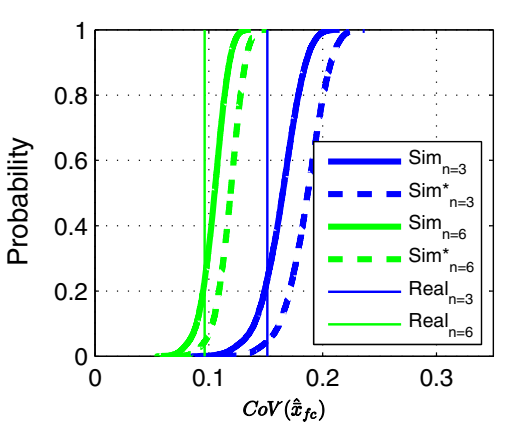

(a)

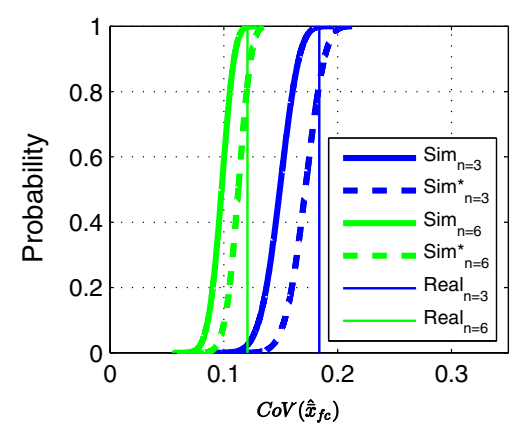

(d)

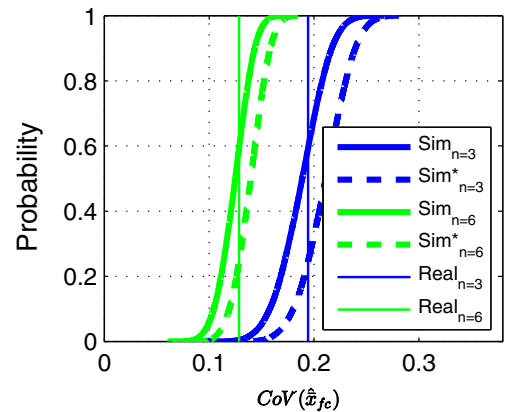

(b)

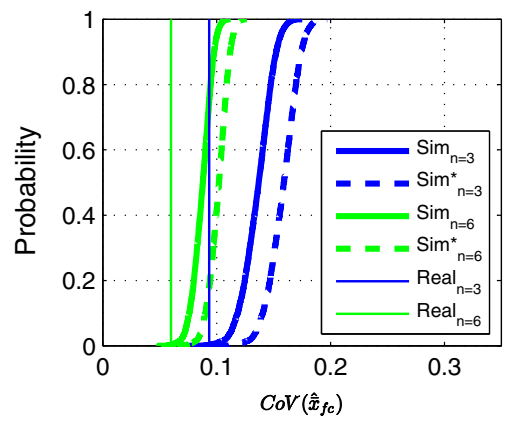

(e)

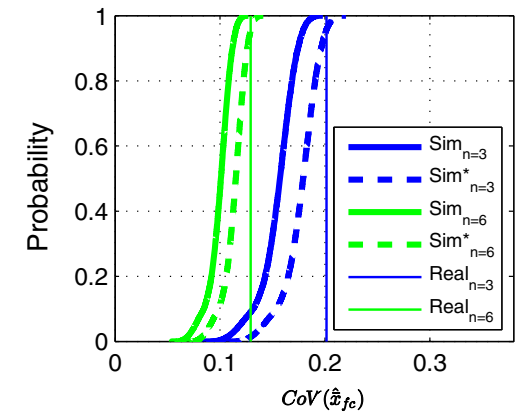

(c)

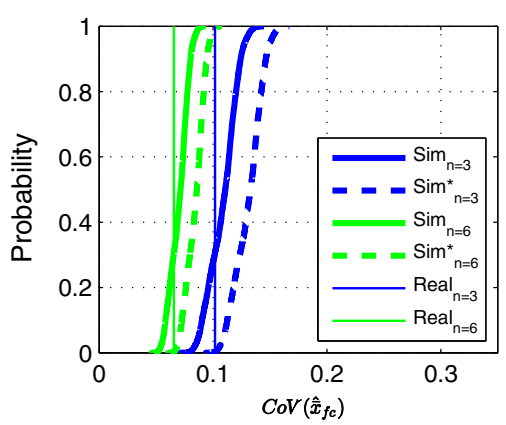

(f)

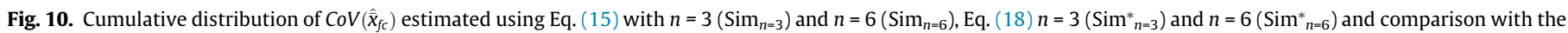
real sampling uncertainty about the mean using 3 (Real ${ }_{n=3}$ ) and 6 (Real ${ }_{n=3}$ ) core samples for datasets (a) C1, (b) C2, (c) C3, (d) C4, (e) C4* and (f) C5.

empirical correlations (i.e. Eqs. (14) and (17)). Consequently, the proposed methods can be improved by adding more data. With respect to the differences between the curves obtained with $n=3$ and $n=6$ cores, it can be seen that, although these approaches provided good results regarding the estimate of the mean concrete strength, the sampling variability almost doubles when the lower sample size is adopted. Therefore, these approaches are not adequate to provide an effective control of the uncertainty in the estimate of the mean value of the concrete strength.

\section{Conclusions}

A finite population statistics-based approach that uses auxiliary information for the assessment of concrete strength in existing RC buildings has been presented in this study. The proposed approach effectively controls the uncertainty in the estimate of the variability of the concrete strength in a population as well as the uncertainty in the estimate of the mean value of the concrete strength. The approach relies on a discretization of the concrete strength distribution within the building considering that a single concrete strength value can be assigned to each structural element, thus making the variability a direct representation of the member-tomember heterogeneity. Other sources of variability such as within member variability and the uncertainty related to the test procedure were excluded from the proposed approach. However, analyzing the importance of these factors is recommended by repeating tests whenever possible. To assess the variability of a finite population of concrete strength values, an empirical model was proposed that correlates the $\mathrm{CoV}$ of concrete core strength values and the $\mathrm{CoV}$ of populations of RN values assessed in the same locations. The adequacy of the proposed empirical model to estimate the $\mathrm{CoV}$ of the concrete strength using indirect measurements of the concrete strength has been shown using five datasets involving core strength results and RN values. These results showed that the proposed method enhances the use of NDTs for the assessment of the concrete strength in existing buildings since it leads to a reduction of the uncertainty in the estimation of the concrete strength variability. It is noted that the empirical model that was developed does not account for test repeatability issues that may affect the regression. Such approach was selected to reflect scenarios where the number of tests that can be carried out is limited.

Regarding the estimate of the mean value of the concrete strength, the simulations carried out within the present study showed that, on average, the mean value of a region can be estimated with an acceptable uncertainty using a number of core compression tests obtained from $15 \%$ to $30 \%$ of the members in the region. However, this approach leads to inadequate estimates of the mean concrete strength variability. The proposed method also provides a better estimate of the mean concrete strength variability, assuming that NDTs can capture the expected variability of the concrete strength of a finite population of structural elements where the concrete strength is assumed to be homogeneous.

In conclusion, the presented study highlights the importance of using auxiliary data provided by NDTs when assessing the concrete compressive strength of an existing building and proposes the use of a strategy based on finite population principles to manage the uncertainty in the estimation of concrete strength statistics.

\section{References}

[1] R. Caspeele, L. Taerwe, Influence of concrete strength estimation on the structural safety assessment of existing structures, Constr. Build Mater. 62 (2014) 77-84.

[2] A. Der Kiureghian, O. Ditlevsen, Aleatory or epistemic? Does it matter?, Struct Saf. 31 (2) (2009) 105-112.

[3] F.M. Bartlett, J.G. MacGregor, Statistical analysis of the compressive strength of concrete structures, ACI Mater. J. 93 (2) (1996) 158-168.

[4] M.T. Cristofaro, A. D’Ambrisi, M. De Stefano, R. Pucinotti, M. Tanganelli, Studio sulla Dispersione dei Valori di Resistenza a Compressione del Calcestruzzo di Edifici Esistenti, Il Giornale delle Prove non Distruttive Monitoraggio e Diagnostica 2 (2012) (in Italian).

[5] Y. Shimizu, M. Hirosawa, J. Zhou, Statistical analysis of concrete strength in existing reinforced concrete buildings, in: Proceedings of 12 th World Conference On Earthquake Engineering, Japan, 2000. 
[6] A. Masi, M. Vona, Estimation of the in-situ concrete strength: provisions of the European and Italian seismic codes and possible improvements, in: E. Cosenza (Ed.), Eurocode 8 Perspectives From the Italian Standpoint Workshop, Doppiavoce, Naples, Italy, 2009, pp. 67-77.

[7] R.C. Drysdale, Variation of concrete strength in existing buildings, Mag. Concr. Res. 25 (85) (1973) 201-207.

[8] M.G. Stewart, Workmanship and its influence on probabilistic models of concrete compressive strength, ACI Mater. J. 92 (4) (1995) 361-372.

[9] A. Masi, L. Chiauzzi, An experimental study on the within-member variability of in situ concrete strength in RC building structures, Constr. Build Mater. 47 (2013) 951-961.

[10] CEN, Eurocode 8: Design of Structures for Earthquake Resistance. Part 3: Assessment and Retrofitting of Buildings, 2005. Brussels.

[11] ASCE, Seismic Evaluation and Retrofit of Existing Buildings (ASCE/SEI 41-13), American Society of Civil Engineers, Reston, Virginia, USA, 2014.

[12] Code of Interventions, Organization for Earthquake Resistant Planning and Protection, Ministry of Environment Planning and Public Works, Greece, 2011.

[13] Circolare 2 febbraio 2009. n. 617 Approvata dal consiglio superiore dei Lavori Pubblici. Istruzioni per l'applicazione delle "Nuove norme tecniche per le costruzioni" di cui al decreto ministeriale 14 gennaio; 2008-2009 [in Italian].

[14] A. Fiore, F. Porco, G. Uva, M. Mezzina, On the dispersion of data collected by in situ diagnostic of the existing concrete, Constr. Build Mater. 47 (2013) 208217.

[15] C. Sarndal, B. Swensson, J. Wretman, Model Assisted Survey Sampling, Springer-Verlag, New York, 2003.

[16] G. Uva, F. Porco, A. Fiore, M. Mezzina, Proposal of a methodology of in situ concrete tests and improving the estimate of the compressive strength, Constr. Build Mater. 38 (1) (2013) 72-83.

[17] F. Jalayer, F. Petruzzelli, I. Iervolino, G. Manfredi, Accounting for the effect of in-situ tests and inspections on the performance assessment of existing buildings, in: Proceedings of the 14th European Conference on Earthquake Engineering, 2010. Ohrid, Macedonia.
[18] X. Chen, S. Wu, J. Zhou, Variability of compressive strength of concrete cores, J. Perform. Constr. Facil. 28 (4) (2014) 06014001.

[19] D. Breysse, Non-destructive evaluation of concrete strength: an historical review and a new perspective by combining NDT methods, Constr. Build Mater. 33 (2012) 139-163.

[20] EN 13791, Assessment of In Situ Compressive Strength in Structures and Precast Concrete Components, European Standard, CEN, 2007.

[21] K. Szilágyi, Rebound Surface Hardness and Related Properties of Concrete PhD dissertation, Budapest University of Technology and Economics, Budapest, Hungary, 2013.

[22] G. Fabbrocino, A. Di Fusco, G. Manfredi, In Situ evaluation of concrete strength for existing constructions: critical issues and perspectives of NDT methods, in: Fib Symposium "Keep Concrete Attractive", 2005. Budapest, Hungary.

[23] M. Brognolli, Prove e controlli non distruttivi per la verifica degli edifici esistenti secondo la normativa sismicae le norme tecniche per le costruzioni, in: Convegno presso l'Ordine degli Ingegneri della Provincia di Brescia, 2007.

[24] D.C. Montgomery, G.C. Runger, Applied Statistics and Probability for Engineers, third ed., Wiley, New York, 2003.

[25] A. Monteiro, A. Gonçalves, Assessment of characteristic strength in structures by the rebound hammer test according to EN 13791:2007, in: Proceedings of NDTCE'09 Conference, 2009. Nantes, France.

[26] S. Biondi, E. Candigliota, In situ tests for seismic assessment of RC structures, in: Proceedings of 14th World Conference on Earthquake Engineering, Beijing, China, 2008.

[27] M.N. Soutsos, D. Breysse, V. Garnier, A. Goncalves, A.V. Monteiro, Estimation of on-site compressive strength of concrete, in: D. Breysse (Ed.), Non-Destructive Assessment of Concrete Structures: Reliability and Limits of Single and Combined Techniques. RILEM State of the Art Reports, Springer, Netherlands, 2012, pp. 119-186.

[28] D. Breysse, J. Martínez-Fernández, Assessing concrete strength with rebound hammer: review of key issues and ideas for more reliable conclusions, Mater. Struct. 47 (2014) 1589-1604. 